\title{
Memoryless Input-Output Encoding for Networked Systems with Unknown Constant Time Delay
}

\author{
Tilemachos Matiakis and Sandra Hirche
}

\begin{abstract}
Communication time delay in a networked control system (NCS) degrades the performance and may lead to instability. The time delay value depends on the network configuration, e.g. number of nodes and is not exactly known during the controller design stage. Based on a novel delayindependent input-output approach the problem of unknown constant time delay is addressed in this paper. The plant and the controller input and output are encoded by a memoryless transformation into new variables which are transmitted over the communication channel. Thereby finite gain $L_{2}$ stability is achieved. The performance of the memoryless encoding approach, validated in simulations and experiments, is superior over two other approaches, the standard small gain and a leadlag control approach.
\end{abstract}

\section{INTRODUCTION}

Due to the increasing complexity of control systems and novel requirements, such as decentralized control, the traditional control architecture with point to point interconnection between the plant and the controller is more and more replaced by networked control systems (NCS). In NCS the components are spatially separated and the control loop is physically closed through a communication network. Significant advantages are reduced wiring and cost, increased modularity, easier maintenance and system diagnosis as well as higher flexibility and reconfigurability, meaning that nodes can be added or removed without additional wiring effort. However, the signal transmission between the plant and the controller in NCS can no longer be regarded ideal. Time delay, data drop out and limited communication resources in the closed loop system represent challenges for the control synthesis, see e.g. [1] for an overview. The transmission characteristics depend on the number of active nodes sharing the communication channel and are typically not exactly known during the controller design stage.

In this work the problem of unknown constant time delay is addressed. It is well-known that time delay in a closed control loop degrades the performance and can lead to instability. Most common in the time delay systems literature are state-space approaches based on a Lyapunovlike stability analysis, see [2], [3] for an overview. In practice, however, the input-output model of a plant is often easier to

T. Matiakis is with the Institute of Automatic Control Engineering, Technische Universität München, D-80290 Munich, Germany t.matiakis@tum.de

S. Hirche is a visiting researcher with Fujita Lab., Dept. of Mechanical and Control Engineering, Tokyo Institute of Technology, 152-8552 Tokyo, Japan s.hirche@ieee.org

This work was supported in part by the Japanese Society for the Promotion of Science (JSPS) by a Postdoctoral Fellowship for Foreign Researchers granted to the second author. obtain than the full state space model. Delay-independent input-output approaches are rarely applied in practice as the necessary small gain condition - the open loop gain less then one - renders these approaches very conservative. For instance, systems with integrators in the open loop cannot be considered, resulting thus in a poor tracking performance to a reference input. The input-output approaches considered in [4], [5] assume the time delay to be known.

As an alternative, in this paper a novel delay-independent input-output approach is investigated. In contrast to standard approaches plant and controller output are not directly sent over the network. Here, the transmitted variables are obtained by encoding plant and controller input and output via a memoryless time-invariant linear transformation, recently introduced in [6]. The approach is applicable to the class of input-feedforward-output-feedback-passive (IFOFP) plants comprising nonlinear, time-varying, and some "well-behaving" unstable input-output relations. As a result of the input-output transformation the IF-OFP property of the plant is preserved through the network independently of the time delay. Any controller-plant pair satisfying a certain finite gain $L_{2}$ stability condition without time delay, is finite gain $L_{2}$ stable also with arbitrarily large constant time delay. The knowledge of the time delay value is not needed for the synthesis of a stable system. Furthermore, the system can be designed such that the transient performance is rather insensitive to the time delay value [6]. This paper addresses performance and design issues. A comparison with two other approaches, the standard delay-independent small gain and a delay-dependent lead-lag control approach, is conducted showing the superior performance of the input-output encoding approach. Experiments with a one degree-of-freedom robotic system convincingly validate the proposed approach.

The remainder of this paper is organized as follows: In Section II. some background is presented, followed by the system description with the input-output transformation in Section III. Performance and design issues are discussed in Section IV. followed by the performance comparison in simulation and experiment in Section V. and conclusions in Section VI.

\section{BACKGROUND}

\section{A. L $L_{2}$ Stability}

Let $\|u\|_{L_{2}}$ denote the $L_{2}$ norm of a piecewise squareintegrable function $u(\cdot): \mathbb{R}_{+} \rightarrow \mathbb{R}^{m}$ with $\mathbb{R}_{+}$being the set of non-negative real numbers and $\mathbb{R}^{m}$ the Euclidean space of dimension $m$. The truncation of $u(\cdot)$ up to the time $t$ is denoted by $u_{t}(\cdot)$, and the extended space of Lebesgue 
integrable functions by $L_{2 e}$. We consider causal input-output mappings $h: u \rightarrow y$ with $h(u(t=0))=0$. Among the variety of input-output stability notions we consider finite gain $L_{2}$ stability in this paper.

Definition 1: A system is said to be finite gain $L_{2}$ stable if there exists a constant $\gamma \geq 0$ such that between each input $u(\cdot) \in L_{2 e}$ and the corresponding output $y(\cdot) \in L_{2 e}$ of the system for each $t \in[0, \infty)$ the following inequality holds

$$
\left\|y_{t}\right\|_{L_{2}} \leq \gamma\left\|u_{t}\right\|_{L_{2}} \text {. }
$$

The smallest possible value $\gamma$ satisfying (1) is the $L_{2}$ gain of the system.

\section{B. Input-Feedforward-Output-Feedback-Passivity}

The feedback components in this paper are assumed to be input-feedforward-output-feedback-passive (IF-OFP).

Definition 2: A dynamical system is said to be IF-OFP if there exist constants $\delta, \varepsilon \in \mathbb{R}$ such that for each input signal $u(\cdot) \in L_{2 e}$ and each $t \in[0, \infty)$ we have [7]

$$
\int_{0}^{t} u^{T}(\tau) y(\tau) \mathrm{d} \tau \geq \delta\left\|u_{t}\right\|_{L_{2}}^{2}+\varepsilon\left\|y_{t}\right\|_{L_{2}}^{2}
$$

In physical interpretation the lefthand term of the inequality represents the external energy flow into the system up to the time $t$. The IF-OFP property is a generalization of the passivity concept. If $\delta=\varepsilon=0$ then the system is passive, i.e. it does not generate energy. If $\delta=0$ and $\varepsilon>0$ the system is called output-feedback strictly passive and if $\delta>0$ and $\varepsilon=0$ input-feedforward strictly passive; in both these cases the system dissipates energy. If one or both of the values $\delta, \varepsilon$ are negative then there is a shortage of passivity in the system. In other words, the system can generate energy, but this energy is bounded by the squared $L_{2}$ norm of the input and/or the output signal. Note, that IF-OFP is a special case of dissipativity with a special quadratic supply rate [8], [9]. The class of IF-OFP systems is quite general and includes non-linear, time-varying and even some inputoutput unstable systems, however, not with finite escape time. Concerning the domain of the constants $\delta, \varepsilon$ the following holds.

Lemma 1: [6] Without loss of generality the domain of $\delta, \varepsilon$ in IF-OFP systems (2) is considered to $\quad$ be $\quad \Omega=\Omega_{1} \cup \Omega_{2} \quad$ with $\quad \Omega_{1}=\{\delta, \varepsilon \in \mathbb{R} \mid \delta \varepsilon<1 / 4\}$ and $\Omega_{2}=\{\delta, \varepsilon \in \mathbb{R} \mid \delta \varepsilon=1 / 4 ; \varepsilon>0\}$.

In case $(\delta, \varepsilon) \notin \Omega$ degenerate cases occur: Either no restriction is imposed on the input-output behavior by (2), or there is no system satisfying (2), see [6] for the proof.

One important stability result for closed loop systems comes from the IF-OFP property of its subsystems. Consider two IF-OFP systems $h_{p}$ and $h_{c}$ satisfying (2) with some $\delta_{i}, \varepsilon_{i}$ with $i \in\{p, c\}$.

Proposition 1: [7] The negative feedback interconnection of $h_{p}$ and $h_{c}$ is finite gain $L_{2}$ stable if

$$
\varepsilon_{c}+\delta_{p}>0 \quad \text { and } \quad \varepsilon_{p}+\delta_{c}>0 .
$$

The proof is given in [7]. Clearly, some of $\delta_{i}, \varepsilon_{i}$ can be negative if compensated by other positive values. Within the passivity formalism this can be interpreted as compensating

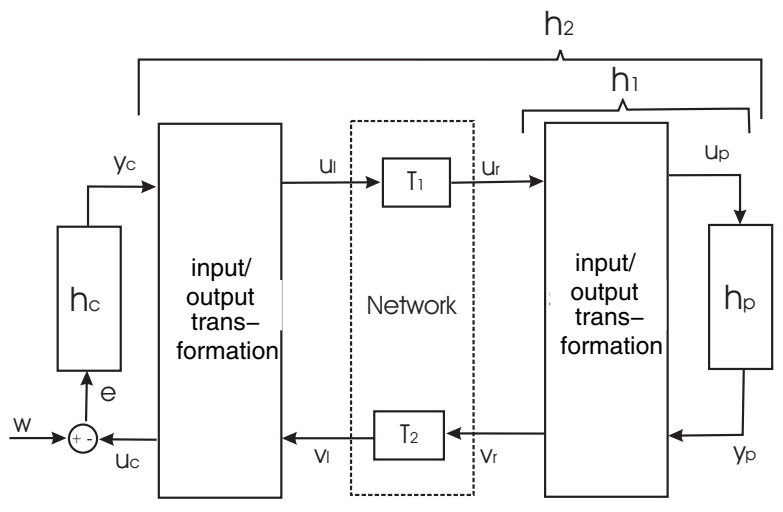

Fig. 1. NCS with input-output encoding (input-output transformation).

shortage of passivity of the one system with the excess of passivity of the other and vice-versa.

\section{Interpretation in the Complex Plane}

For single-input-single-output (SISO) stable linear timeinvariant (LTI) systems with the transfer function $G(s)$ there is a geometrical interpretation of the IF-OFP property and finite gain $L_{2}$ stability in the complex plane. If $\varepsilon>0$ the Nyquist plot of a stable IF-OFP system lies in a circle with center point $R=\frac{1}{2 \varepsilon}$ and radius $r=\frac{\sqrt{1-4 \varepsilon \delta}}{2 \varepsilon}$; if $\varepsilon<0$ it lies everywhere on the complex plane except for a circle with center $R=\frac{1}{2 \varepsilon}$ and radius $r=-\frac{\sqrt{1-4 \varepsilon \delta}}{2 \varepsilon}$. If $\varepsilon=0$ then $\operatorname{Re}\{G(j \omega)\} \geq \delta$ holds, i.e. the Nyquist plot lies in the plane right of $\delta$. For a passive system $\delta=\varepsilon=0$ the IF-OFP circle coincides with the right half plane as $R \rightarrow \infty, r \rightarrow \infty$ holds. Analogously, finite gain $L_{2}$ stability with gain $\gamma$ can be seen as a limit case of IF-OFP with $\delta=-\varepsilon \gamma^{2}, \varepsilon \rightarrow \infty$ from (2), hence $R=0, r=\gamma$, i.e. the finite gain $L_{2}$ circle where the Nyquist plot is confined to, has its center at the origin and a radius $r=\gamma$.

For interpretation of later results the Linear Fractional Transformation (LFT) is used. The LFT is a conformal mapping of the complex plane $\mathbb{C} \cup \infty$ to itself, defined by the relation

$$
\text { LFT }: z \rightarrow \frac{c_{1} z+c_{2}}{c_{3} z+c_{4}}
$$

where $c_{1}, c_{2}, c_{3}, c_{4} \in \mathbb{C}$. If $c_{1} c_{4}-c_{2} c_{3} \neq 0$ it is bijective, otherwise it is a constant, i.e. $z \rightarrow \frac{c_{1}}{c_{3}}=\frac{c_{2}}{c_{4}}$. Main characteristic of the LFT is that it maps circles on the complex plane either to circles or to straight lines. Similarly, the LFT maps lines in the complex plane either to lines or to circles.

\section{INPUT-OUtPUt ENCODING APPROACH}

Most generally, MIMO systems with the same dimension $m$ of input and output can be considered as plant and controller in this approach. However, for the ease of notation a SISO plant and controller are considered, i.e. $m=1$. Where non-ambiguous, the time argument $t$ is dropped for convenience of notation.

\section{A. System Description and Assumptions}

The plant is described by a mapping $y_{p}=h_{p}\left(u_{p}\right)$ from the plant input $u_{p} \in L_{2 e}$ to the plant output $y_{p} \in L_{2 e}$, the controller by a mapping $y_{c}=h_{p}(e)$ from the control error $e \in L_{2 e}$ 
to the controller output $y_{c} \in L_{2 e}$, see Fig. 1. The control error is defined as $e=w-u_{c}$ with $w \in L_{2 e}$ the desired value and $u_{c}$ the lefthand side output of the communication channel. The plant is connected to the controller through a communication network. However, instead of directly transmitting plant (controller) output over the communication channel, a linear combination of plant (controller) output and input is sent to the corresponding receiver side. Similarly to [6] a memory-less transformation is favored due to the limited computational power on the plant side. The transformation matrix $M \in \mathbb{R}^{2 \times 2}$ acts on the plant and the controller inputoutput vector $z_{p}^{T}=\left[u_{p} y_{p}\right]$ and $z_{c}^{T}=\left[y_{c} u_{c}\right]$, respectively

$$
s_{r}=M z_{p} \quad \text { and } \quad s_{l}=M z_{c},
$$

where $s_{r}^{T}=\left[u_{r} v_{r}\right]$ and $s_{l}^{T}=\left[u_{l} v_{l}\right]$ represent the values transmitted over the communication channel, see Fig. 1. The transformation matrix $M$ is parameterized as a rotation matrix $R$ and a scaling matrix $B$

$$
\begin{aligned}
& M=R B \quad \text { with } \\
& R=\left[\begin{array}{rr}
\cos \theta & \sin \theta \\
-\sin \theta & \cos \theta
\end{array}\right] ; \quad B=\left[\begin{array}{cc}
\sqrt{b} & 0 \\
0 & \frac{1}{\sqrt{b}}
\end{array}\right],
\end{aligned}
$$

with the rotation angle $\theta \in\left[-\frac{\pi}{2}, \frac{\pi}{2}\right]$ and the scaling parameter $b>0$, both constant. The mapping by $M$ is a bijection; it belongs to the class of special linear transformations, i.e. $\operatorname{det} M=1$, hence is non-singular, an inverse exists. Note that for $M=I_{2}$, with $I$ the identity matrix, the standard approach without transformation is recovered.

The network is modelled with a forward time delay $T_{1}$ (plant to controller channel) and backward time delay $T_{2}$. The value $u_{l}$ is transmitted from the controller to the plant side arriving as value $u_{r}(t)=u_{l}\left(t-T_{1}\right)$. Accordingly for the backward channel $v_{l}(t)=v_{r}\left(t-T_{2}\right)$ holds. It is assumed that $u_{r}(t)=0 \quad \forall t \in\left[0, T_{1}\right)$ and $v_{l}(t)=0 \forall t \in\left[0, T_{2}\right)$. For further reference, the following subsystems: $v_{r}=h_{1}\left(u_{r}\right)$ and $u_{c}=h_{2}\left(y_{c}\right)$ are defined, see also Fig. 1.

Throughout the paper we assume that the closed loop system is well posed, i.e. for each input signal $w \in L_{2 e}$ there exists a unique solution for the signals $e, u_{c}, y_{c}, u_{l}, v_{l}, u_{r}, v_{r}, u_{p}, y_{p}$ that causally depends on $w$. Further, we assume the following system properties:

1) The time delays $T_{1}, T_{2}$ are arbitrarily large but constant.

2) Plant $h_{p}$ and controller $h_{c}$ are IF-OFP with $\delta_{i}, \varepsilon_{i}$ with $i \in\{p, c\}$ satisfying Proposition 1, i.e. the feedback interconnection without time delay is finite gain $L_{2}$ stable.

\section{B. Stability}

In the following the most important stability results are briefly reviewed, for a detailed analysis, proofs and further discussion refer to [6]. For the following consideration the closed loop system is decomposed into the negative feedback interconnection of subsystem $h_{2}$, and the controller $h_{c}$, see Fig. 1. By proper selection of the transformation angle $\theta$ the subsystem $h_{2}$ is IF-OFP with exactly the plant IF-OFP parameters $\delta_{p}, \varepsilon_{p}$ independently of time delay.
Theorem 1: [6] The subsystem $h_{2}$ is IF-OFP with $\delta_{p}, \varepsilon_{p}$ if and only if the following holds

$$
\cot 2 \theta=\varepsilon_{p} b-\frac{\delta_{p}}{b}
$$

and

$$
\sin (\theta) \cos (\theta)-\frac{\delta_{p}}{b} \cos ^{2}(\theta)-\varepsilon_{p} b \sin ^{2}(\theta) \geq 0 .
$$

The second condition (7) merely defines one of the two existing solutions of $\theta$ in (6). As this solution exists for each $b>0$, this scaling parameter can be chosen freely to meet performance requirements.

From this result it is straightforward to conclude finite gain $L_{2}$ stability of the closed loop system.

Corollary 1: [6] The closed loop system with the transformation (5) and the transformation parameters satisfying Theorem 1 is finite gain $L_{2}$ stable independently of the time delay.

Crucial point of the proposed approach is that the inputoutput transformation transforms the IF-OFP plant $h_{p}$ into a finite gain $L_{2}$ stable subsystem $h_{1}$ such that

$$
\left\|v_{r, t}\right\|=\left\|h_{1}\left(u_{r, t}\right)_{t}\right\| \leq \gamma_{h_{1}}\left\|u_{r, t}\right\| \quad \forall t ; \quad \gamma_{h_{1}} \geq 0,
$$

holds. The derivation of the gain $\gamma_{h_{1}}$ can be found in [6]. A constant time delay operator does not alter this $L_{2}$ gain $\gamma_{h_{1}}$ during transmission, since it has a $L_{2}$ gain equal to one. The inverse transformation $M^{-1}$, i.e. the decoding, is a bijection as well, and therefore the exact IF-OFP property $\delta_{p}, \varepsilon_{p}$ of the plant is recovered at the subsystem $h_{2}$. As an important result, the feedback interconnection of any controller-plant pair satisfying the finite gain $L_{2}$ condition from Proposition 1 without time delay is finite gain $L_{2}$ stable for arbitrary large time delay by using the proposed encoding approach.

An intuitive geometrical interpretation can be given for a LTI system. The input-output transformation represents a LFT, see Section II-C, from the plant transfer function $G_{p}(j \omega)$ to the transfer function $G_{1}(j \omega)$ of the subsystem $h_{1}$

$$
G_{1}(j \omega)=\frac{\cos (\theta) G_{p}(j \omega)-b \sin (\theta)}{\sin (\theta) G_{p}(j \omega)+b \cos (\theta)}
$$

as straightforward derivable from the transformation equations (4). This LFT maps the circle that the Nyquist plot of the IF-OFP plant $G_{p}(j \omega)$ lies in, to a finite $L_{2}$ gain circle with center at the origin, see Fig. 2. The arbitrary large time delay in series connection with $G_{1}$ does not alter this circle as it only rotates each point around the origin as observable from the transfer function $G_{1}(j \omega) e^{-j \omega T}$ where $T=T_{1}+T_{2}$. The inverse transformation, since it is a bijection, maps the finite $L_{2}$ gain circle back to the initial IF-OFP circle of the plant. The IF-OFP property of the plant is restored to the subsystem $h_{2}$ independently of the time delay.

Remark 1: In case of an unstable plant, the plant is locally pre-stabilized with the right hand input-output transformation. This is clear from the fact that each IF-OFP plant results in a finite gain $L_{2}$ stable system after the right hand transformation as observable from (8).

Remark 2: In case of a passive plant the LFT (9) with $\theta=45^{\circ}$ recovers the well-known scattering transforma- 
(a)

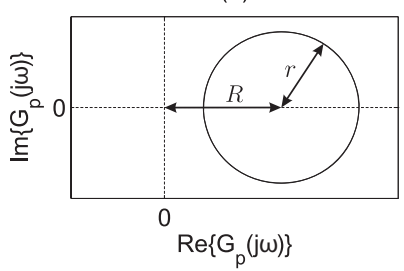

(b)

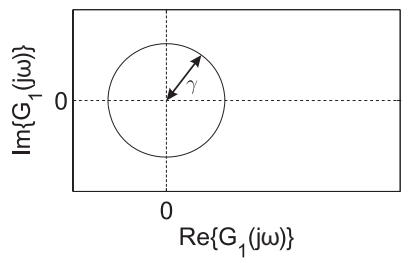

Fig. 2. (a) Nyquist plot area of the IF-OFP plant $G_{p}$. (b) Nyquist plot area of the finite gain $L_{2}$ stable subsystem $G_{1}(s)$.

tion widely used for the stabilization of teleoperation systems in the presence of arbitrarily large constant time delay [10], [11]. The scattering transformation maps the right half plane to the unit circle, i.e. a passive system to a finite gain $L_{2}$ stable system with $L_{2}$ gain one.

Remark 3: The input-output encoding makes the closed loop systems stability unaffected by any causal operator with a $L_{2}$ gain $\gamma \leq 1$ inserted within the loop of encoded variables. In fact, many scattering based approaches to cope with communication effects such as time-varying delay [12] and packet loss [13], [14] are based on the small gain principle within the scattering variable domain. These approaches are straightforward to apply here.

\section{Performance and Design Issues}

In the following some performance issues; the closed loop system is assumed to be finite gain $L_{2}$ stable.

\section{A. Steady State Behavior and Zero Delay Case}

The steady state behavior with input-output encoding and time delay is equivalent to the one without input-output encoding and without time delay, as in the steady state $s_{l}=s_{r}$ holds, hence $z_{c}=M^{-1} s_{l}=M^{-1} s_{r}=M^{-1} M z_{p}=z_{p}$. The same, $z_{c}=z_{p}$, holds in the zero delay case, meaning that the system reduces to that without time delay and without input-output encoding; nominal performance is achieved.

In terms of steady state error the proposed approach clearly outperforms the standard small gain approach. The standard small gain approach, e.g. for LTI systems, requires $\left|G_{c}(j \omega) G_{p}(j \omega)\right|<1, \forall \omega$, i.e. free integrators in the open loop are not allowed. This leads to a rather large steady state error, e.g. $|e(t)|_{t \rightarrow \infty}>\frac{1}{2}|w|$ for a reference step input $w$. In the proposed approach free integrators in plant or controller do not necessarily contradict Corollary 1 . This can be easily seen using counterexamples, e.g. $G_{p}(s)=\frac{s+1}{s+2}$ and the stabilizing controller $G_{c}(s)=1+\frac{1}{s}$ where the values of $\delta, \varepsilon$ are easily defined from the Nyquist plot. Thus, steady state error zero and independent-of-delay stability can be guaranteed simultaneously. Further, in [6] it has been shown that the transformation and the controller can be designed such that the closed loop performance is rather insensitive to different time delay values.

\section{B. Design Procedure}

Based on the stability result from the previous section a controller design procedure is proposed.

1) Define the plant $\delta_{p}, \varepsilon_{p}$ values, (e.g. Nyquist plot).

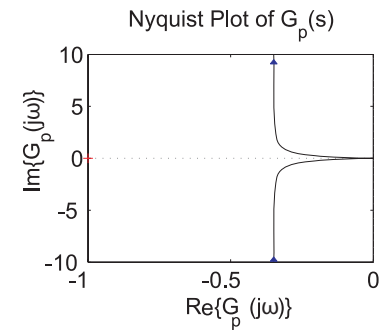

Fig. 3. Nyquist plot of the plant transfer function $G_{p}(j \omega)$.

2) From Theorem 1 define the minimum values for $\delta_{c}, \varepsilon_{c}$ of the controller so as to guarantee stability for the closed loop system.

3) Find a controller and a value for the scaling parameter $b$ of the transformation, so as to satisfy performance criteria under the constraints of $\delta_{c}, \varepsilon_{c}$ and the angle $\theta$ of the transformation given according to Theorem 1 .

In step 1) it is desirable to determine the tightest bound for $\delta_{p}, \varepsilon_{p}$ in order to avoid conservatism. However, this is non-trivial in general. How to define the optimal controller with respect to some performance criteria in step 3) is still an open problem and subject of current research.

\section{PERFORMANCE COMPARISON}

In order to show the efficacy of the input-output encoding approach a comparison is performed with a controller satisfying the classical small gain approach for delay-independent stability, and with the delay-dependent lead-lag controller. It should be mentioned that the latter comparison is unfair since delay-dependent methods usually lead to less conservative results. In lack of a systematic controller design methodology with input-output encoding a general performance comparison is difficult at this stage. Instead, a case study is conducted here with the controller designed through numerical optimization. A one degree-of-freedom robotic system later used for experimental validation is considered with the approximated transfer function

$$
G_{p}(s)=\frac{36}{s^{2}+10.15 s} .
$$

\section{A. Controller Design}

For fair comparison the controller for all approaches are designed by numerical optimization using the integrated squared tracking error

$$
J=\int_{0}^{t_{f}} e^{2}(\tau) \mathrm{d} \tau,
$$

as cost function. The numerical optimization is performed for a nominal time delay of $T=300 \mathrm{~ms}$ over a horizon of $t_{f}=5 \mathrm{~s}$ using fmincon of the Matlab optimization toolbox. As basic structure for the controller a lead-lag element

$$
G_{c}(s)=k \frac{s+a}{s+c},
$$

is considered where $k, a, c>0$ are parameters to be determined by numerical optimization. The exact design procedure for all three cases is explained in the next. 
1) Input-Output Encoding: Following the design steps given in Section IV-B, first the values $\delta_{p}, \varepsilon_{p}$ are determined from the Nyquist plot of (10) presented in Fig. 3. Clearly, $\operatorname{Re}\left\{G_{p}(j \omega)\right\}>-0.3494$, further $\operatorname{Im}\left\{G_{p}(j \omega)\right\} \rightarrow \infty$ as $\omega \rightarrow 0$ giving $\delta_{p}=-0.3494, \varepsilon_{p}=0$, see Section II-C. For stability based on Theorem $1 \delta_{c}>0$ and $\varepsilon_{c}>0.35$ must be satisfied for the controller. The Nyquist plot of the lead-lag controller is a circle with center on the real axis at $R$ and radius $r$ given by

$$
R=\frac{k(a+c)}{2 c} ; \quad r=\frac{k|a-c|}{2 c} .
$$

From these values, using the equations from Section II-C, follows $\delta_{c}=\frac{R^{2}-r^{2}}{2 R}>0$, which is always satisfied as long as $k, a, c>0$. Furthermore, (11) and the requirement $\varepsilon_{c}>0.35$ leads to

$$
\frac{k(a+c)}{2 c}<1.4286
$$

imposing a stability constraint to the lead-lag controller parameter values. The resulting constrained optimization problem can be formulated as $\min _{k, a, c, b, \theta} J$ subject to the equality constraint (6), and the inequality constraints (7), (12), and $k, a, c, b>0$. The numerical optimization gives the controller for the input-output transformation

$$
G_{t r}(s)=\frac{1.6160(s+2.5833)}{s+3.3628},
$$

with all constraints satisfied and $b=0.1816, \theta=13.73^{\circ}$. The optimal cost function value is $J=0.2927$.

2) Classical Small Gain Controller: In order to achieve delay-independent stability without input-output encoding the small gain condition has to be satisfied requiring $\left|G_{0}(j \omega)\right|_{\infty}=\left|G_{p}(j \omega) G_{s g}(j \omega)\right|_{\infty}<1 \quad \forall \omega$ with $G_{s g}$ the small gain controller transfer function and $\left|G_{0}(j \omega)\right|_{\infty}$ the $H_{\infty}$ norm of the open loop transfer function $G_{0}$. As the small gain condition cannot be satisfied with the integrator in the plant (10) a high pass component is added to the lead-lag controller. Further, the controller gain $k$ is fixed to $k=1 /\left|G_{0}(j \omega)\right|_{\infty}$. The optimization problem is given by $\min _{a, c} J$ subject to the inequality constraint $a, c>0$. The numerical optimization gives the controller

$$
G_{s g}=\frac{0.1855(s+5.3574)}{(s+3.5258)} \frac{s}{0.001 s+1},
$$

where the latter part represents the high pass component. The optimal cost function value is $J=1.1192$.

3) Delay-Dependent Lead-Lag Controller: Similarly to the previous case the optimization problem is represented by $\min _{k, a, c} J$ subject to the constraint $k, a, c>0$ resulting in the controller

$$
G_{l l}=\frac{2.6136(s+2.9028)}{(s+14.3436)},
$$

with the optimal cost function value $J=0.2917$.

\section{B. Simulations}

The performance is not only compared for the nominal time delay, but for different values as well, in order to
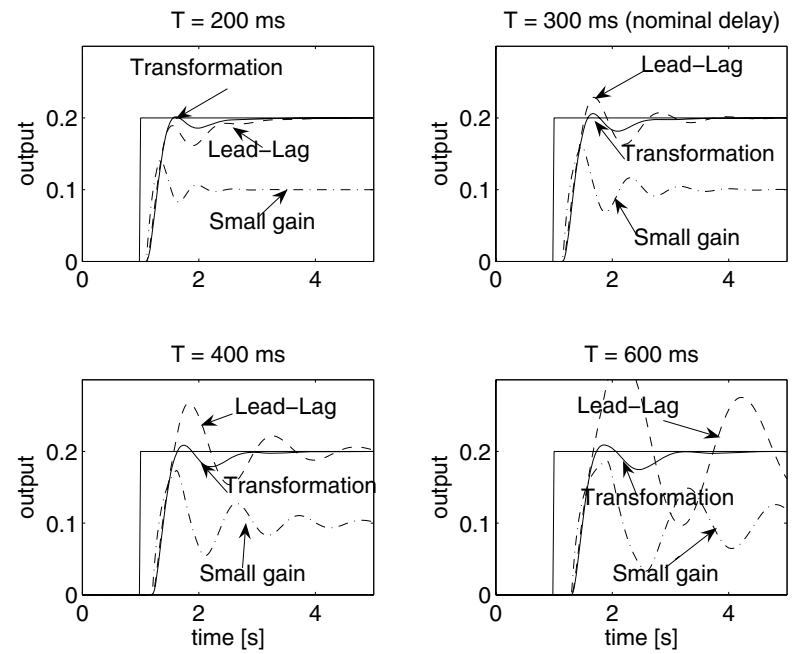

Fig. 4. Simulation results: Step response with input-output encoding (transformation), small gain based controller and lead-lag controller for various time delay values

TABLE I

Simulation Results: $\pm 5 \%$ Settling Time

\begin{tabular}{|c||c||c||c||c|}
\hline Time Delay $(\mathbf{m s})$ & $\mathbf{2 0 0}$ & $\mathbf{3 0 0}$ & $\mathbf{4 0 0}$ & $\mathbf{6 0 0}$ \\
\hline \hline I/O Encoding & $1.18 \mathrm{~s}$ & $1.36 \mathrm{~s}$ & $1.53 \mathrm{~s}$ & $1.85 \mathrm{~s}$ \\
\hline lead-lag & $1.32 \mathrm{~s}$ & $1.53 \mathrm{~s}$ & $3.11 \mathrm{~s}$ & - \\
\hline Small gain & $1.05 \mathrm{~s}$ & $1.87 \mathrm{~s}$ & $3.38 \mathrm{~s}$ & - \\
\hline
\end{tabular}

validate the low sensitivity of the closed loop performance with input-output encoding to different time delay values as indicated in [6]. A step input of magnitude $0.2 \mathrm{rad}$ and round trip time delay values $T \in\{200,300,400,600\} \mathrm{ms}$ are considered.

From the simulation results in Fig. 4 we observe that the input-output (I/O) encoding approach outperforms the other approaches, not only for the nominal time delay, but also for the other time delay values. The overshoot is small and only slightly affected by the time delay value; the settling time is much smaller than with the other two approaches and does not change significantly for different time delay as well, see also Table I. The small gain controller gives a steady state error of approximately $50 \%$ in all cases. With increasing time delay the settling time grows significantly. The lead-lag controller without input-output encoding performs well at the nominal time delay but the performance is, as expected, sensitive to the time delay value: Still stable for the maximum time delay value tested it shows significant overshoot and settling time when the time delay increases.

\section{Experiments}

The experimental testbed consists of the 1DOF pendulum shown in Fig. 5, connected to a PC running under RT Linux. The original design of the pendulum can be found in [15]. The DC-motor current, resulting in a torque, is provided by the PWM amplifier operated under current control. The reference signal is given by a voltage from the $\mathrm{D} / \mathrm{A}$ converter output of the I/O board. The position of the lever is measured by an optic pulse incremental encoder and processed by 


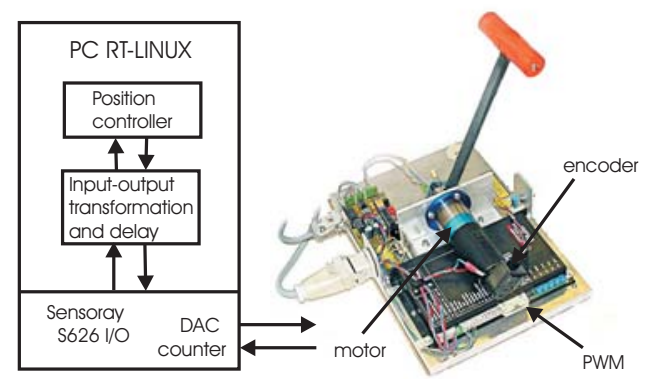

Fig. 5. Experimental testbed with one degree-of-freedom robotic system.
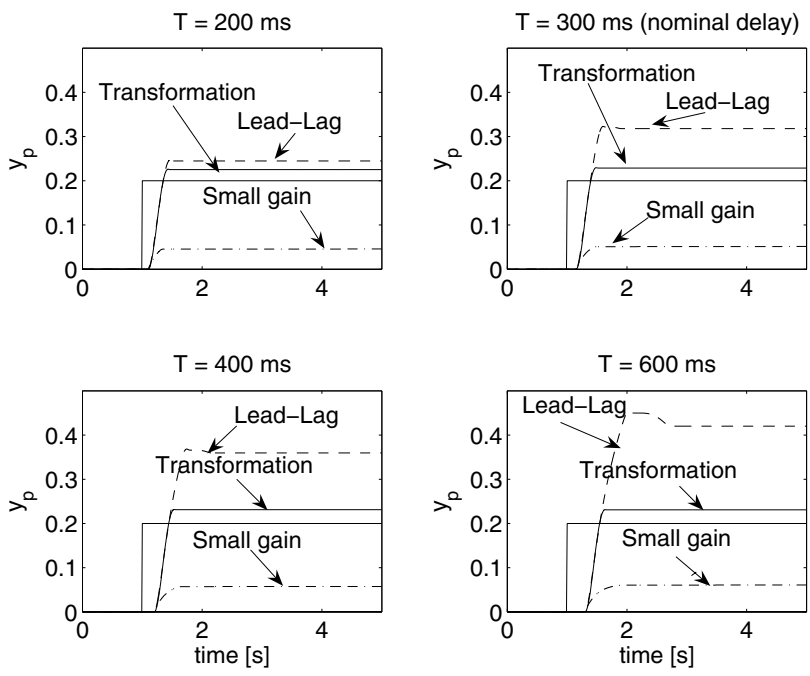

Fig. 6. Experimental results: Step response with input-output encoding (transformation), small gain based controller and lead-lag controller for various time delay values.

a quadrature encoder on the I/O board. The control loop including the controller, the input-output transformation, and the time delays are implemented in MATLAB/SIMULINK blocksets. Standalone realtime code is generated directly from the SIMULINK model. The experiments are performed with a sampling time interval $T_{A}=1 \mathrm{~ms}$.

The experimental results are presented in Fig. 6. The proposed approach outperforms even the delay-dependent leadlag controller for nominal time delay. Not only the smallest steady state error appears, see also Table II, the performance is furthermore rather insensitive to the time delay value. The small gain controller has, as in the simulations, a very high steady state error. The steady state error with the delaydependent lead-lag controller is high as well and increases with increasing time delay. The observed steady state error in all cases is most probably due to unmodelled nonlinearities such as friction and backlash. In summary, the proposed input-output encoding approach is convincingly validated.

TABLE II

EXPERIMENTAL RESUlTS: STEADY STATE ERROR IN \%

\begin{tabular}{|c||c||c||c||c|}
\hline Time Delay $(\mathbf{m s})$ & $\mathbf{2 0 0}$ & $\mathbf{3 0 0}$ & $\mathbf{4 0 0}$ & $\mathbf{6 0 0}$ \\
\hline \hline Transformation & 12.6 & 14.4 & 15.5 & 15.4 \\
\hline lead-lag & 20.8 & 46 & 81.9 & 109.9 \\
\hline Small gain & 77.1 & 74.3 & 71.3 & 69.6 \\
\hline
\end{tabular}

\section{Vi. Conclusions}

A novel input-output approach for NCS guaranteeing finite gain $L_{2}$ stability independently of the constant time delay is investigated in this paper. Input and output of plant and controller are encoded into new variables which are transmitted over the communication channel. The performance is superior over the classical delay-independent input-output approach with direct transmission of the plant (controller) output employing the small gain theorem. Even a delaydependent input-output approach is outperformed in a case study based on simulations and experiments. Clearly the input-output encoding approach holds great promise for NCS. Future work is to develop a systematic control design methodology.

\section{ACKNOWLEDGMENTS}

The authors are grateful to Martin Buss for his helpful advice and support. The helpful comments of the anonymous reviewers are appreciated.

\section{REFERENCES}

[1] Y. Tipsuwan and M. Y. Chow, "Control methodologies in network control systems," Control Engineering Practice, vol. 11, pp. 10991011, 2003.

[2] J.-P. Richard, "Time-delay systems: an overview of some recent advances and open problems," automatica, vol. 39, pp. 1667-1694, 2003.

[3] K. Gu, V. Kharitonov, and J. Chen, Stability of Time-Delay Systems, B. Boston, Ed., 2003.

[4] T. Georgiou and M. Smith, "Robust Stabilization in the Gap Metric: Controller Design for Distributed Plants," IEEE Transactions on Automatic Control, vol. 37, no. 8, pp. 1133-1143, August 1992.

[5] C. Bonnet and J. Partington, "Bezout Factors and L1-Optimal Controllers for Delay Systems using a Two-Parameter Compensator Scheme," IEEE Transactions on Automatic Control, vol. 44, no. 8, pp. 1512-1521, August 1999.

[6] T. Matiakis, S. Hirche, and M. Buss, "A Novel Input-OutputTransformation Method to Stabilize Networked Systems Independentof-Delay," in 17th International Symposium on Mathematical Theory of Networks and Systems, MTNS2006, Kyoto, Japan, 2006, to appear.

[7] H. K. Khalil, Nonlinear Systems, P. Hall, Ed., 1996.

[8] J. C. Willems, "Dissipative Dynamical Systems - Part I: General Theory," Arch. Rational Mechanics Analysis, vol. 45, pp. 321-351, 1972.

[9] — , "Dissipative Dynamical Systems - Part II: Linear Systems with Quadratic Supply Rates," Arch. Rational Mechanics Analysis, vol. 45, pp. 352-393, 1972.

[10] R. J. Anderson and M. W. Spong, "Bilateral control of teleoperators with time delay," IEEE Transactions on Automatic Control, vol. 34, no. 5, pp. 494-501, May 1989.

[11] G. Niemeyer and J. Slotine, "Stable adaptive teleoperation," International Journal of Oceanic Engineering, vol. 16, no. 1, pp. 152-162, 1991.

[12] R. Lozano, N. Chopra, and M. Spong, "Passivation of Force Reflecting Bilateral Teleoperators with Time Varying Delay," in Proceedings of the 8. Mechatronics Forum, Enschede, Netherlands, 2002, pp. 954962.

[13] B. Berestesky, N. Chopra, and M. W. Spong, "Discrete Time Passivity in Bilateral Teleoperation over the Internet," in Proceedings of the IEEE International Conference on Robotics and Automation ICRA'04, New Orleans, US, 2004, pp. 4557-4564.

[14] S. Hirche and M. Buss, "Packet Loss Effects in Passive Telepresence Systems," in Proceedings of the 43rd IEEE Conference on Decision and Control, Paradise Island, Bahamas, 2004, pp. 4010-4015.

[15] H. Baier, M. Buss, F. Freyberger, J. Hoogen, P. Kammermeier, and G. Schmidt, "Distributed pc-based haptic, visual and acoustic telepresence system-experiments in virtual and remote environments," in Proceedings of the IEEE Virtual Reality Conference VR99, 1999, pp. $118-125$. 